\title{
DEFINITION IN TERMS OF ORDER ALONE IN THE LINEAR CONTINUUM AND IN WELL-ORDERED SETS*
}

\author{
BY \\ OSWALD VEBLEN \\ First definition of the continuum.
}

A linear continuum is a set of elements $\{\boldsymbol{P}\}$ which we may call points, subject to a relation $<$ which we may read precedes, governed by the following conditions, $A$ to $E$ :

\section{A. General Postulates of Order.}

1. $\{P\}$ contains at least two elements.

2. If $P_{1}$ and $P_{2}$ are distinct elements of $\{P\}$ then either $P_{1}<P_{2}$ or $P_{2}<P_{1}$.

3. If $P_{1}<P_{2}$ then $P_{1}$ is distinct from $P_{2}$. $\dagger$

4. If $P_{1}<P_{2}$ and $P_{2}<P_{3}$, then $P_{1}<P_{3}$.

\section{B. Dedekind Postulate of Closure. $\ddagger$}

If $\{P\}$ consists entirely of two infinite subsets, $\left[P^{\prime}\right]$ and $\left[P^{\prime \prime}\right]$ such that $P^{\prime}<P^{\prime \prime}$, then there is either a $P_{0}^{\prime}$ of $\left[P^{\prime}\right]$ such that $P^{\prime}<P_{0}^{\prime}$ whenever $P^{\prime} \neq P_{0}^{\prime}$ or a $P_{0}^{\prime \prime}$ of $\left[P^{\prime \prime}\right]$ such that $P_{0}^{\prime \prime}<P^{\prime \prime}$ whenever $P^{\prime \prime} \neq P_{0}^{\prime \prime}$.

\section{Pseudo-archimedean postulate.}

1. If $\{P\}$ is an infinite set then there exists a subset $\left[P_{\nu}\right]$, such that $P_{\nu}<P_{\nu+1}(\nu=1,2,3, \ldots)$ and if $P$ is any element of $\{P\}$ for which $P_{1}<P$, then there exists a $\nu$ such that $P<P_{\nu}$.

* Presented to the Society December 30, 1904, uuder the title, Non-Metrical Definition of the Linear Continuum. Received for pablication January 26, 1905.

$\dagger$ The propositions $A_{3}$ and $A_{4}$ say that $<$ is "non-reflexive" and "transitive." It follows immediately that it is "non-symmetric," for if $P_{1}<P_{2}$ and $P_{2} \prec P_{1}$ could occur simultaneously, 4 would lead to $P_{1}<P_{1}$, contrary to 3 .

$\ddagger$ Usually called a postulate of continuity; but a set would hardly be called continuous unless it also satisfied condition $D$. 
2. If $\{P\}$ is an infinite set then there exists a subset $\left[P_{\nu}^{\prime}\right]$, such that $P_{\nu+1}^{\prime}<P_{\nu}^{\prime}(\nu=1,2,3, \cdots)$ and if $P$ is any element of $\{P\}$ for which $P<P_{1}^{\prime}$, then there exists a $\nu$ such that $P_{\nu}^{\prime}<P$.

\section{Postulate of Density.}

If $P_{1}$ and $P_{3}$ are any two elements of $\{P\}$ such that $P_{1}<P_{3}$ then there exists an element $P_{2}$ of $\{P\}$ such that $P_{1}<P_{2}$ and $P_{2}<P_{3}$.

Definition. An element $P_{2}$ such that $P_{1}<P_{2}<P_{3}$ is said to lie between $P_{1}$ and $P_{3}$. If $P_{1}$ and $P_{3}$ have an element between them the set of all such elements is called the segment $P_{1} P_{3}$. Thus the segment $P_{1} P_{3}$ does not contain either $P_{1}$ or $P_{3}$ which are called its end-points. A segment plus its end-points is called an interval. One segment is said to contain another if it contains all its elements.

\section{E. Postulate of uniformity.}

For every element $P$ of a set $\{P\}$ which satisfies conditions $A, B, C, D$, and for every $\nu(\nu=1,2,3, \ldots)$ there exists a segment $* \sigma_{\nu p}$ such that the set of segments $\left[\sigma_{\nu P}\right]$ has the following properties $\dagger$ :

1. For a fixed $P, \sigma_{\nu P}$ contains $\sigma_{v+1 P}$.

2. For a fixed $P, P$ lies on every $\sigma_{\nu P}$ and is the only such element.

3. For every segment $\tau$, there exists $a \nu, \nu_{\tau}$, such that for no $P$ does $\sigma_{\nu_{\tau} P}$ contain $\tau$.

\section{Relations of the above to other definitions.}

Of the above postulates or items of the definition, those grouped under the heading $A$ constitute the usual definition of order which probably received its first explicit formulation in the hands of G. Cantor. They can easily be shown equivalent to the postulates given by other writers $\ddagger$ in terms of the relations of "precedence" or " betweenness" or "separation." The proof of this equivalence and the deduction of the elementary theorems of order are here omitted since they are covered fairly well by the literature cited in the footnote above.

From conditions $A$ and $B$ follow the theorems that every bounded set has a

* The notation $\sigma_{\nu P}$ indicates that $\sigma_{\nu P}$ is a function both of $v$ and of $P$.

$\dagger$ A metrical special case of a set $\left[\sigma_{\nu P}\right]$ is obtained by letting $\sigma_{\nu P}$ be a segment of length $1 / \nu$ whose middle point is $P$. It is evident that as $\nu$ increases the segment $\sigma_{\nu P}$ closes down on the points $P$ in the uniform manner described in number 3 above. The theorem of uniform continuity involves consideration of a set like $\left[\sigma_{\nu p}\right]$.

$\ddagger$ Cf. footnote on page 88 of this volume; and also E. V. Huntington, $A$ set of postulates for real algebra, comprising postulates for a one-dimensional continuum and for the theory of groups, pages. 17-41 of this volume. 
least upper and greatest lower bound, * that every infinite bounded set has at least one limiting element, and also the important.

Heine-Borel Theorem. If every element of an interval $P_{1} P_{2}$ belongs to at least one segment $\sigma$ of a set of segments $[\sigma]$, then there exists a finite subset of $[\sigma], \sigma_{1}, \sigma_{2}, \cdots, \sigma_{n}$, such that every element of $P_{1} P_{2}$ belongs to at least one of the segments $\sigma_{1}, \sigma_{2}, \ldots, \sigma_{n}$. This theorem, as well as a generalization of it, is proved in the Bulletin of this society, vol. 10, p. 436, by a method which applies to our hypotheses. We may note in passing, what seems to be a new result, that, since conditions $A$ and $B$ are satisfied by any well-ordered set in the sense of Cantor, the Heine-Borel theorem is a true theorem of any wellordered set.

Conditions $A$ and $B$ are sufficient for a large portion of the theory of functions of a real variable. Condition $D$ provides that every element of $\{P\}$ shall be a limiting element. The three conditions $A, B, D$ are common to all current definitions of the continuum in so far as they involve the notion of order.

At this point there have been three essentially different modes of advance. The first, which we may call analytical, introduces a symbol for the combining of any two elements to produce a third, i. e., regards the elements as numbers. $\dagger$ The second, a geometrical method, brings in the notion of congruence of segments. Either of these schemes provides a relation between segments at different positions in the continuum, thus affording a basis for theorems of uniformity, e. g., uniform continuity. The third method, that of G. CANTor, provides for this uniformity by postulating the presence of an everywhere dense subset ordinally similar to the ordinary rational numbers. CantoR's definition $\ddagger$ is equivalent to $A, B, D$, a condition that the continuum shall have two endpoints, and the following :

$F .\{P\}$ contains a subset $\{\boldsymbol{P}\}$ of cardinal $\boldsymbol{\aleph}_{0}(i$. e., an enumerable subset $)$ such that between every two elements of $\{\boldsymbol{P}\}$, there is an element of $\{\widetilde{P}\}$.

Cantor's definition is simpler than the other two in that relations of congruence or operations of addition and multiplication do not appear among his undefined symbols. On the other hand he introduces the notion of infinite cardinal number. The definitions furnished by our conditions $A, B, C, D, E$ are stated entirely in terms of the relation $<$ and the general logical symbols that appear in every mathematical discussion. While the language in which our

* An upper bound of a set $[x]$ is an element, $B$, such that $x<B$ for every $x \neq B$. A least upper bound is an upper bound $B_{0}$ such that if $B$ is any other upper bound of $[x], B_{0} \prec B$. $A$ limiting element of a set $[x]$ is an element $A$ such that every segment which contains $A$ contains all element of $[x]$ different from $A$.

† Cf. E. V. Huntington, loc. cit.

$\ddagger$ G. Cantor, Zur Begriindung der transfiniten Mengenlehre, I, Math ematisobe Annalen, vol. $46(1895)$, p. 510. 
condition $E$ is stated may be a little more complicated than that used in the other definitions, it is often more convenient of application.*

Cantor has proved, loc. cit., p. 510, that any two sets satisfying his conditions are in a one-to-one correspondence preserving order relations, $i$. e., that his definition is categorical. $\dagger$ Therefore, to show the same for our definition, we need only to show that $F$ is a consequence of our postulates. Among other results of this theorem it may be remarked that, since a continuum according to our definition is in one-to-one reciprocal correspondence with the continuum defined by the usual scheme of deriving irrational numbers from rationals, we have (by mediation of the correspondence) a definition of addition and multiplication in terms of the relation $<\cdot \ddagger$

\section{Proof of condition $F$.}

Let $P_{1} P_{1}^{\prime}$ be any interval of the continuum and let $\left[\sigma_{v p}^{\prime}\right]$ be that subset of the set $\left[\sigma_{v p}\right]$ referred to in postulate $E$ for which the subscript point $P$ lies on the interval $P_{1} P_{1}^{\prime}$. The subset of $\left[\sigma_{v p}^{\prime}\right]$ obtained by fixing a value of $\nu_{1}$ is an infinite set such that every point $P$ of $P_{1} P_{1}^{\prime}$ lies on one segment $\sigma_{v p}^{\prime}$. By the HeINe-Borel theorem, there is a finite subset of this infinite set having the same property. The end-points of the segments of this set are a finite set of points

$$
P_{\nu}^{1} P_{\nu}^{2} \ldots P_{\nu}^{m_{\nu}}
$$

The set of all such points $\left[P_{\nu}^{m_{\nu}}\right], \nu=1,2, \ldots$, is evidently enumerable and by the third item in condition $E$, has a point between any two points of the interval $P_{1} P_{1}^{\prime}$.

Consider now the sets of points $\left[P_{\nu}\right]$ and $\left[P_{\nu}^{\prime}\right]$ of postulate $C$. There evidently exists an integer $n$ such that

$$
P_{n}^{\prime}<P_{n} .
$$

* Compare, for example, Theory of plane curves in non-metrical analysis situs, these Transac. tions, vol. 6 (1905), p. 83.

$\dagger$ The word "cateyorical" implies that between any two classes $\{P\}$ and $\{Q\}$ satisfying the conditions named, there is a one-to-one correspondence such that if $P_{1} \prec P_{2}$ then for the corresponding elements of $\{Q\}, Q_{1}<Q_{2}$. It therefore also implies that any proposition stated in terms of the relation $\prec$ either is true for all classes satisfying our conditions or is false for all such classes. Cf. vol. 5, p. 346 of these Transactions and also some remarks of E. V. HunTINGTON in his paper on Complex Algebra in the present number.

$\ddagger$ An interesting situation is obtained by introducing a postulate of uniformity among the hypotheses of plane analysis situs (ct. p. 84 of this volume). If the postulate is applied to the straight line, the line is necessarily a continuum but it is not obvious that other curves are. If it is applied to the plane, the segments $\sigma_{\nu p}$ in this case being triangular regions, all continuous curves are continua, but it is not obvious that there is a one-to-one reciprocal correspondence between the plane and a set of number pairs. There seems thus to be possible a still more general formulation of the RIEMANN-HELMHOLZ-LiE problem of the foundations of geometry than that obtained by HILBERT. 
The enumerable set of intervals $P_{n+k}^{\prime} P_{n+k}, k=1,2, \ldots$, is evidently such that for any point $P$, there is a value of $k$ such that $P$ lies on $P_{n+k}^{\prime} P_{n+k} \cdot$ On each of these segments there is, by the paragraph above, an enumerable everywhere dense set of points. The points of the enumerable set, so obtained, of enumerable everywhere dense sets of points constitute an enumerable and everywhere dense set as required by the conclusion of $F$.

\section{Independence of the postulates.}

The independence of the items of conditions $A$ is proved by the examples, $A_{1}, A_{2}, A_{3}, A_{4}$ given on pages 89-90 of this volume of the Transactions. The independence of condition $B$ is proved by example $B_{2}$ and of condition $D$ by example $B_{1}$ on the same page.

To prove the independence of condition $C_{1}$, we observe that a well-ordered set of type $+\Omega$ (the set of all well-ordered sets of cardinal number $\aleph_{0}$ ) does not satisfy condition $C$. For if a sequence such as $\left[P_{\nu}\right]$ should exist the set of all elements preceding each $P_{\nu}$ would be enumerable and hence $\Omega$ itself would be enumerable, contrary to CANTOR's theorem. If each element of this set and its next following element are connected by a set similar to the continuum of real numbers greater than zero and less than one, the resulting set satisfies conditions $A, B, D, E$ ( $E$ vacuously), but not $C_{1}$. The independence of $C_{2}$ is proved by employing $* \Omega$ instead of $\Omega$ as above.

To prove the independence of $E$, consider the set of all pairs of real numbers $(x, y),-\infty<x<+\infty, 0 \leqq y \leqq 1$, the relation $<$ being defined so that

$$
\begin{aligned}
& \left(x_{1} y_{1}\right)<\left(x_{2} y_{2}\right) \text { when } x_{2} \neq x_{1} \text { if } x_{1}<x_{2}, \\
& \left(x_{1} y_{1}\right)<\left(x_{2} y_{2}\right) \text { when } x_{2}=x_{1} \text { if } y_{1}<y_{2} .
\end{aligned}
$$

That $A, C, D$ are satisfied is evident; $B$ is also seen easily if the Dedekind postulate is applied first to the variable $x$ and then to $y$. If $E$ were satisfied, $F$, which is a consequence of it, would also be satisfied; but an everywhere dense subset would have to include a point for every value of $x$, whereas $[x]$ is not enumerable.

\section{Postulates for well-ordered sets.}

A set of independent postulates defining the general notion, well.ordered set, is obtained by taking together, with conditions $A$ and $B$, the following:

$W_{1}$. The set $\{\boldsymbol{P}\}$ contains an element $\boldsymbol{P}_{0}$ such that fur no element $\boldsymbol{P}^{\prime}$ docs $P^{\prime}<P_{0}$.

$P_{0}$ is called the first element.

† See Cantur, loc. cit., p. 207. 
$W_{2}$. If $P_{1}$ is any element of $\{P\}$ for which there are elements $P^{\prime}, P_{1}<P^{\prime}$, $\{P\}$ contains an element $P_{2}$ such that $P_{1}<P_{2}$ and such that for no element $P^{\prime \prime}$ do there exist both of the relations,

$$
P_{1}<P^{\prime \prime} \text { and } P^{\prime \prime}<P_{2},
$$

$P_{2}$ is called the element next following $P_{1}$, and $P_{1}$ the element next preceding $P_{2}$.

There is no difficulty in seeing that conditions $W_{1}, W_{2}$, and $B$ are equivalent to the conditions of Cantor's definition of a well-ordered set.* CAntor defines particular classes of well-ordered sets by means of their cardinal numbers. This can also be done purely ordinally. For example, a finite set is one which satisfies conditions $A, B, W_{1}, W_{2}$, together with $W_{3}$ and $W_{4}$.

$W_{3}$. Every element of $\{P\}$ except $P_{0}$ hxs a next preceding element.

$W_{4}$. The set $\{P\}$ contains an element $\bar{P}$ such that for no element $P^{\prime}$ does $I^{-}<P^{\prime}$

$\bar{I}$ is called a last element.

A set of type $\omega$ is determined by conditions $A, B, W_{1}, W_{2}, W_{3}$, together with $W_{\omega}$, which is the negative of $W_{4}$.

$W_{\omega}$. If $P$ is any element of $\{\boldsymbol{P}\},\{\boldsymbol{P}\}$ contains an element $\boldsymbol{P}^{\prime}$ such that $P<P^{\prime}$.

A set of the type associated with the number-class $Z\left(\boldsymbol{N}_{0}\right)$, i. e., the type of set which Cantor defines by prescribing the cardinal $\boldsymbol{N}_{v}$, is given by conditions $A, B, W_{1}, W_{2}$, together with $W_{3^{\prime}}$, and $W_{4^{\prime}}$ :

$W_{3^{\prime}}$. Every element of $\{P\}$ except $P_{0}$ has a next preceding element or is the least upper bound of a subset of type $\omega$ (as just defined).

$W_{4^{\prime}} \cdot\{P\}$ either contains a last element or a subset $\left\{P_{\nu}\right\}$ of type $\omega$ such thut for every $P$, there exists a $\nu_{P}$ for which $P<P_{\nu_{P}}$.

The proof may readily be given that a set satisfying these conditions is enumerable. To define the type $\Omega$ we take $A, B, W_{1}, W_{2}, W_{3^{\prime}}$ together with $W_{\Omega}$ which is the exact negative of $W_{t^{\prime}}$, i. e., with "neither" substituted for "either" and "nor" for "or." To define the type of sets associated with the number-class $Z\left(\mathbf{N}_{1}\right)$, we take $A, B, W_{1}, W_{2}$ together with $W_{3^{\prime \prime}}$ and $W_{4 "}$.

$W_{3^{\prime \prime}}$. Every element of $\{P\}$ except $P_{0}$ has a next preceding element or is the lectst upper bound of a subset of type $\omega$ or $\Omega$.

$W_{4^{\prime \prime}} \cdot\{P\}$ either contains a last element or a subset [Q] of type $\omega$ or $\Omega$ such that for every $P$ there exists a $Q_{P}$ for which $P<Q_{P}$.

'This scheme of definition is evidently adequate to determine any class of wellorilered sets.

\footnotetext{
*CANTor, Beiträge zur Begrïndung der transfiniten Mengenlehre, Mathematische Annalen, vol. 49 (1898), p. 207.
} 
Modification of the definition of the continuum.

By the following analysis, the postulate of uniformity can be made formally a little weaker. Adopting the language of Cantor, let us denote by the words fundamental sequence a subset of $\{P\}$ of type $\omega$ - that is, a subset $\left\{P_{\kappa}\right\}$, $\kappa=1,2,3, \ldots$, where $P_{\kappa}<P_{\kappa+1}$. Any element which is the least upper bound of a fundamental sequence is a principal element. Postulate $E$ may now be replaced by the following two, $E^{\prime}$ and $E^{\prime \prime}$.

$E^{\prime}$. Let $\left[P^{\prime}\right]$ be the set of all principal elements of $\{P\}$; if any such exist, there exists a set of sets of points $\left[\left\{P_{\nu}\right\}_{P^{\prime}}\right]$ such that:

1. If $P^{\prime}$ is any point of $\left[P^{\prime}\right]$, the set $\left\{P_{\nu}\right\}_{P^{\prime}}$ has $P^{\prime}$ as least upper bound and is such that $P_{v}<P_{v+1}$.

2. If $P^{\prime \prime}$ and $P^{\prime \prime \prime}\left(P^{\prime \prime}<P^{\prime \prime \prime}\right)$ are any two points of $\left[P^{\prime}\right]$, there is a value $\nu_{1}$ of $\nu$ such that for no point $P^{\prime} d_{0}$ there exist the relations

$$
P_{\nu_{1}}<P^{\prime \prime}, P^{\prime \prime \prime}<P^{\prime},
$$

if $P_{\nu_{1}}$ belongs to the set $\left\{P_{\nu}\right\}_{P^{\prime}}$

$E^{\prime \prime}$. Every ele:nent of $\{P\}$ is a principal element.

The proof that condition $F$ is a consequence of $A, B, C, D, E$ needs to be modified when $E$ is replaced by $E^{\prime}$ and $E^{\prime \prime}$. By $E^{\prime \prime}$, the set $\left[P^{\prime}\right]$ of $E^{\prime}$ is identical with $\{P\}$. For a fixed value of $\nu$, the segments $P_{\nu} P=\sigma_{\nu P}$, where $P_{v}$ belongs to the set $\left\{P_{v}\right\}_{P}$, constitute a set to each segment of which, every point of $\{P\}$ is either an interior or an end point.

Fixing attention on a particular interval $P_{1} P_{1}^{\prime}$ of the continuum, for a fixed value of $\nu$ its points fall into two classes, those which are interior points of segments $\sigma_{v P}$ (for $P$ on $P_{1} P_{1}^{\prime}$ ) and those which are end points of such segments without being interior to any. Call this latter set $\left[\bar{P}^{\nu}\right]$.

Defining the relation $>$ so that $P_{1}>P_{2}$ if and only if $P_{2}<P_{1}$, we easily see that with respect to the sense $>,\left[\bar{P}^{\nu}\right]$ is a well ordered set of which $P_{1}^{\prime}$ is the first element. But in view of condition $E^{\prime \prime}$ it satisfies conditions $W_{3^{\prime}}$ and $W_{t^{\prime}}$ as well as $A, B, W_{1}, W_{2} . \quad\left[\bar{P}^{\nu}\right]$ is therefore enumerable, and its points are the upper end points in the sense $<$ of an enumerable set of intervals which include all points of $P_{1} P_{1}^{\prime}$ as interior or end points. By applying the HeIneBuREL theorem to these intervals separately, we obtain, instead of a finite set as when using condition $E$, a numerably infinite set

$$
P_{\nu}^{1}, P_{\nu}^{2}, \ldots P_{\nu}^{m_{\nu}} \ldots \text {. }
$$

The set $\left[P_{,{ }^{\prime \prime}}\right], \nu=1,2,3 \ldots$ is enumerable and everywhere dense on $P_{1} P_{1}^{\prime}$, and the rest of the argument proceeds as in the former case. 